\title{
Role of spectroscopic factors in the potential-model description of the ${ }^{7} \mathrm{Be}(p, \gamma){ }^{8} \mathrm{~B}$ reaction
}

\author{
Attila Csótó \\ Department of Atomic Physics, Eötvös University, Pázmány Péter sétány 1/A, H-1117 Budapest, Hungary
}

(August 30, 1999)

\begin{abstract}
In standard potential-model descriptions of the ${ }^{7} \mathrm{Be}(p, \gamma){ }^{8} \mathrm{~B}$ reaction the ${ }^{7} \mathrm{Be}+p$ spectroscopic factors $\mathcal{S}$ appear in the cross section. We argue that the microscopic substructure effects which are represented by $\mathcal{S}$ are short-ranged and cannot affect the asymptotic normalization of the wave function. We believe that the standard way of describing reactions in a potential model may be incorrect and the low-energy cross section should not depend on $\mathcal{S}$ in the case of external capture reactions, like ${ }^{7} \mathrm{Be}(p, \gamma)^{8} \mathrm{~B}$
\end{abstract}

PACS number(s): 25.40.Lw, 26.65.+t, 21.60.Gx, 27.20.+n

Recently the ${ }^{7} \mathrm{Be}(p, \gamma)^{8} \mathrm{~B}$ radiative capture reaction has been studied extensively both experimentally and theoretically. This interest is rooted in the fact that the ${ }^{8} \mathrm{~B}$ produced by this reaction in our sun is the main source of the high-energy solar neutrinos [1]. The high-energy solar neutrino flux is directly proportional to the lowenergy $\left(E_{\mathrm{cm}}=20 \mathrm{keV}\right)$ astrophysical cross section factor, $S_{17}(E)$, of ${ }^{7} \mathrm{Be}(p, \gamma)^{8} \mathrm{~B}$. Among the recent experimental results are a new direct measurement of the low-energy cross section by using a ${ }^{7} \mathrm{Be}$ target [2], the determination of $S_{17}(E)$ from the inverse process, the Coulomb dissociation of ${ }^{8} \mathrm{~B}$ [3], and the utilization of transfer reactions in order to determine the asymptotic normalization constant of the bound-state ${ }^{8} \mathrm{~B}$ wave function [4,5], which in turn can be used to extract $S_{17}(0)$. On the theoretical side, the capture reaction has been studied recently in ${ }^{7} \mathrm{Be}+p$ potential models [6].7], in three-body models [8], in shell-models [9], and in microscopic cluster models 10,11. Interesting results came also from the R-matrix study of the experimental data [12], from the investigation of the energy-dependence of $S(E)$ 13], and from the studies of the asymptotic normalization constants of the ${ }^{8} \mathrm{~B}$ wave function 14,15 . Yet, despite all these and other advances, $S_{17}(0)$ is still the most uncertain input parameter in solar models 16 .

In the present work we would like to clarify a few points of this problem in connection with the potential models.

At solar energies the ${ }^{7} \mathrm{Be}(p, \gamma)^{8} \mathrm{~B}$ reaction takes place deep below the Coulomb barrier. This means that the capture cross section receives contributions almost exclusively from those parts of the initial scattering and final bound state wave functions that describe large ${ }^{7} \mathrm{Be}-p$ separations. At low energies the scattering wave functions are almost fully known, as the phase shifts practically coincide with the hard core phase shifts. The asymptotic behavior of the bound state wave function is also known, as it is proportional to the CoulombWhittaker function,

$$
\chi_{I}^{\text {bound }}(r)=\bar{c}_{I} W_{-\eta, l+1}^{+}(k r) / r, \quad r \rightarrow \infty,
$$

where $\eta$ is the Coulomb parameter, $l$ is the relative an- gular momentum between ${ }^{7} \mathrm{Be}$ and $p$, and $I=1,2$ is the channel spin, which comes from the coupling of the ${ }^{7} \mathrm{Be}$ spin and the spin of the proton. Therefore, the zeroenergy ${ }^{7} \mathrm{Be}(p, \gamma){ }^{8} \mathrm{~B}$ cross section depends only on the asymptotic normalization constants $\bar{c}_{I}$ [17,14]. Using a generic formula, which is specified later for the various models, this means

$$
S_{17}(0)=N\left(\bar{c}_{1}^{2}+\bar{c}_{2}^{2}\right) \mathrm{eVb} .
$$

(We note, that the different notations followed in various papers are slightly confusing. Our $\bar{c}$ quantity is the equivalent of $\beta$ in Ref. [14], while the spectroscopic factor $\mathcal{S}$, see below, is denoted by $\mathcal{J}$ there.) We would like to emphasize that we use the Eq. (1) definition of the asymptotic normalization constant in all cases. In the case of a potential-model description, $\chi$ is the so-called single-particle wave function, while in the case of a microscopic model, $\chi$ is the wave function describing the relative motion between ${ }^{7} \mathrm{Be}$ and $p$.

The precise value of $N$ depends on the details of the scattering wave function. In the case of our scattering state coming from the microscopic cluster model [10], which corresponds roughly to $r_{c}=2.4 \mathrm{fm}$ hard-sphere radius [13], $N$ is 37.8 , therefore

$$
S_{17}(0)^{\text {micr. }}=37.8\left(\bar{c}_{1}^{2}+\bar{c}_{2}^{2}\right) \text { eVb. }
$$

Note that in Refs. 10 the integration of the cross section was not done to a sufficiently large radius. All $S_{17}(0)$ values given there should be increased by roughly $0.4 \mathrm{eVb}$. Note also that in Ref. [6] the hard-core scattering states, used in a potential model, were chosen to be in sync with those coming from Refs. 10], but once again the integration distance was too short. Thus, the corrected $S_{17}(20 \mathrm{keV})=37.2\left(\bar{c}_{1}^{2}+\bar{c}_{2}^{2}\right) \mathrm{eVb}$ relation found there, is in agreement with Eq. (3).

The peripheral nature of the ${ }^{7} \mathrm{Be}(p, \gamma)^{8} \mathrm{~B}$ reaction is illustrated in Fig. 1. A schematic local potential is shown between ${ }^{7} \mathrm{Be}$ and $p$. One can see that at $20 \mathrm{keV}$, which is the most effective reaction energy in our sun, the proton 
hits the Coulomb barrier at about $250 \mathrm{fm}$. It has to tunnel through a huge barrier in order to allow the capture to take place. Therefore, the cross section is really sensitive almost exclusively to the asymptotic parts of the wave functions. One can also see in Fig. 1 that the asymptotic normalization of the bound state wave function is most sensitive to the radius of the ${ }^{7} \mathrm{Be}-p$ potential. A slightly bigger radius (shown by the long-dashed line in Fig. If for the sake of illustration the change in the radius is strongly exaggerated) leads to a smaller and narrower barrier, and thus to a significantly larger tunneling probability, which gives a larger cross section [10,18].

In Eqs. (2-3) there is one point which is not yet specified, namely the full normalization of the bound-state wave function. In the case of an 8-body model of the reaction, the full 8-body wave function should be normalized to unity. However, the ${ }^{7} \mathrm{Be}-p$ relative motion wave functions, as one-dimensional functions which contain the $\bar{c}$ constants, are obviously not normalized the same way (see below). In the case of a potential model, the effects of the internal structure of ${ }^{7} \mathrm{Be}$, which are neglected in the model, has to be taken into account in some implicit way. Conventionally this is done through the spectroscopic factors $\mathcal{S}$. The spectroscopic amplitude functions, $g$, of the ${ }^{7} \mathrm{Be}+p$ configuration in ${ }^{8} \mathrm{~B}$ are given [19] as

$$
g(\mathbf{r})=\left\langle\Psi_{\mathbf{r}} \mid \Psi^{8} \mathrm{~B}\right\rangle
$$

where $\Psi^{8} \mathrm{~B}$ is the normalized antisymmetrized 8-body wave function of ${ }^{8} \mathrm{~B}$, while $\Psi_{\mathbf{r}}$ is defined as

$$
\Psi_{\mathbf{r}}=\mathcal{A}\left[\Phi^{7}{ }^{7} \Phi^{p} \delta(\mathbf{r}-\boldsymbol{\rho})\right] .
$$

Here $\mathcal{A}$ is the intercluster antisymmetrizer between ${ }^{7} \mathrm{Be}$ and $p, \Phi^{7} \mathrm{Be}$ and $\Phi^{p}$ are the normalized antisymmetrized internal wave function of ${ }^{7} \mathrm{Be}$ and a spin-isospin eigenstate of the proton, respectively, and $\boldsymbol{\rho}$ is the relative coordinate between ${ }^{7} \mathrm{Be}$ and $p$. The quantum numbers carried by $g$, like the channel spin $I$, the angular momentum coupling, etc., are not indicated here for simplicity. The spectroscopic factor is given as

$$
\mathcal{S}=\int|g(\mathbf{r})|^{2} d \mathbf{r}
$$

This quantity is a measure of the cluster substructure effects which are neglected in a potential model, and can be calculated using a microscopic model, like the shell model or the cluster model, or can be extracted from nuclear reaction measurements.

The various quantities that are calculated from potential models, like the decay widths of resonances, cross sections, etc., contain $\mathcal{S}$ in order to take into account the effects of the neglected microscopic substructure. In other words, the norm of the potential-model wave function is assumed to be different from unity, depending on how large the neglected microscopic effects are. In potential models, the ${ }^{7} \mathrm{Be}(p, \gamma)^{8} \mathrm{~B}$ cross section contains $\mathcal{S}$ and thus the Eqs. (2- -3) expressions are modified as

$$
S_{17}(0)^{\text {pot. }}=37.8\left(\bar{c}_{1}^{2} \mathcal{S}_{1}+\bar{c}_{2}^{2} \mathcal{S}_{2}\right) \mathrm{eVb}
$$

(here the same hard-core scattering state is assumed as in the microscopic model), where $\mathcal{S}_{1}$ and $\mathcal{S}_{2}$ are the channelspin spectroscopic factors. It is important to note that this way of taking into account the microscopic effects relies on the assumption that these effects can be handled separately from the calculation of the matrix element of the cross section. We note also, that most of the potential-model calculations generate the $I=1$ and $I=2$ channel wave functions of the ${ }^{8} \mathrm{~B}$ ground state separately, not in a correct coupled-channel description. In any case, the total single-particle wave function (containing both the $I=1$ and $I=2$ components) is assumed here to be normalized to unity.

We would like to argue, however, that we believe that the conventional definition of the cross section in the potential model might be incorrect. The effects of the microscopic substructure, which leads to the appearance of the spectroscopic factors in the cross section formula, are short-distance corrections because they originate from short-range effects, like the antisymmetrization. Therefore, these effects should only affect the internal parts of the wave functions and should not modify the asymptotic normalization constants. To illustrate this, in Fig. 2 we show the ${ }^{7} \mathrm{Be}-p$ relative-motion wave function $\chi$ of ${ }^{8} \mathrm{~B}$ in the $I=2$ channel, coming from the microscopic cluster model of Refs. 10] (dashed line). The MN effective nucleon-nucleon interaction was used and the total wave function contained only $\left({ }^{4} \mathrm{He}+{ }^{3} \mathrm{He}\right)+p$ terms with cluster size parameters $\beta=0.4 \mathrm{fm}^{-2}$. Note that this relative-motion wave function is the one behind the antisymmetrizer in the ${ }^{8} \mathrm{~B}$ wave function, thus its norm, which is not one, has no physical meaning. Also shown in Fig. 2 are the spectroscopic amplitude $g(r)$ (solid line) defined in Eq. (4), and the Coulomb-Whittaker function of the ${ }^{7} \mathrm{Be}-p$ relative motion multiplied by the asymptotic normalization constant $\bar{c}_{2}=0.763$ (for $I=1$, $\left.\bar{c}_{1}=0.302\right)$, coming from the model (dotted line). The spectroscopic amplitude was calculated using the procedure discussed in Ref. [20]. As one can see, the relative motion function $\chi$ and the spectroscopic amplitude $g$ coincides beyond $r \approx 7 \mathrm{fm}$. The difference between the two functions in the internal region gives a measure of the antisymmetrization effect.

The effect of taking into account the microscopic substructure in the potential model would be similar (although much smaller, because the norm of $g$ is close to one) on the potential-model wave function. Therefore, it seems to us that the usual way of treating microscopic effects in the potential model, through the spectroscopic factors, cannot be right. Multiplying the potential-model wave function by $\sqrt{\mathcal{S}}$ modifies it not only in the internal region but asymptotically as well. We suggest that 
the correct way to take into account the effects of the microscopic substructure in the potential model would be to use the spectroscopic amplitude functions in the expressions of the various matrix elements. In certain cases where the internal parts of the wave functions play the major role, like the decay widths of resonances or the cross sections of non-peripheral reactions, the results would be close to those coming from the conventional definition, because of the Eq. (6) relation. However, in certain cases, like the present peripheral reaction cross section, where only the asymptotic parts of the wave functions are important, there would be no effect coming from the difference between the wave function and $g$. If our suggestion turns out to be correct, then the Eq. (7) cross section formula for potential models should not contain the spectroscopic factors.

We realize of course that our present arguments are rather heuristic. A thorough study of the connection between microscopic and macroscopic approaches to capture reactions, similar to that presented in Ref. [19] for nuclear structure, would be highly welcome.

Using the spectroscopic amplitudes, one of which is shown in Fig. 2, we calculated the spectroscopic factors predicted by our present cluster model. They are $\mathcal{S}_{I_{7}, I}^{J}=$ $\mathcal{S}_{3 / 2,2}^{2}=0.915$ and $\mathcal{S}_{3 / 2,1}^{2}=0.205$. Here $I_{7}$ and $I$ are the spin of ${ }^{7} \mathrm{Be}$ and the channel spin, respectively, while $J$ is the total spin of the ${ }^{8} \mathrm{~B}$ ground state. The spectroscopic factor of the state which contains the excited state of ${ }^{7} \mathrm{Be}\left(I_{7}=1 / 2\right)$ is $\mathcal{S}_{1 / 2,1}^{2}=0.25$ in our model. The total spectroscopic factor corresponding to $I_{7}=3 / 2$ and $J=2$ is $\mathcal{S}=\mathcal{S}_{3 / 2,2}^{2}+\mathcal{S}_{3 / 2,1}^{2}=1.12$, in good agreement with the shell-model predictions of Ref. [6]. We note that the spectroscopic factors given in Refs. 12,21] erroneously do not take into account the $A /(A-1)=8 / 7$ center-ofmass correction factor 22]. The corrected numbers are $\mathcal{S}=1.177,1.166$, and 1.143 for the CK, B, and K shellmodel interactions, again in good agreement with Ref. [6].

We calculated the spectroscopic factors also for the other models and interactions used in Refs. [10] and found them to be between 1.07 and 1.12. One can observe a correlation between $S_{17}(0)$ and $\mathcal{S}$ if the peak position of $g$ is roughly the same: a larger $\mathcal{S}$ leads to a slight increase of $g$ in the asymptotic region, and thus to a bigger $S_{17}(0)$. All our results with the $\mathrm{MN}$ and $\mathrm{MHN}$ interactions fit into this trend. In the case of the V2 interaction the peak position of $g$ is shifted to a slightly larger radius, which makes the corresponding $\mathcal{S}=1.07$ 1.1 small, compared to the large $S_{17}(0)=28.8-29.8 \mathrm{eVb}$ cross section, predicted by the V2 force. We note that our spectroscopic factors are slightly smaller than, but compatible with the shell-model result, $\mathcal{S}=1.1-1.2$ [6]. A conservative estimate of $\mathcal{S}$, based on the shell model and the cluster model, could be around 1.05-1.2.

In summary, we presented circumstantial evidences which indicate that the conventional way of treating microscopic substructure effects in potential models may be wrong. We believe that the correct way of handling those effects would be the use of the spectroscopic amplitude functions, instead of the potential-model wave functions and spectroscopic factors. This would lead to a zero-energy cross section for peripheral reactions, which is independent of the spectroscopic factors.

\section{ACKNOWLEDGMENTS}

This work was supported by OTKA Grants F019701, F033044, and D32513, and by the Bolyai Fellowship of the Hungarian Academy of Sciences. Some preliminary investigations related to the present work were benefited from discussions with R. G. Lovas and K. Varga.

[1] J. N. Bahcall, Neutrino astrophysics (Cambridge University Press, Cambridge, 1989).

[2] F. Hammache et al., Phys. Rev. Lett. 80, 928 (1998).

[3] Motobayashi et al., Phys. Rev. Lett. 73, 2680 (1994); N. Iwasa et al., Journ. Phys. Soc. Japan 65, 1256 (1996); T. Kikuchi et al., Eur. J. Phys. 3, 213 (1998).

[4] W. Liu et al., Phys. Rev. Lett. 77, 611 (1996).

[5] A. Azhari et al., Phys. Rev. Lett. 82, 3960 (1999).

[6] B. A. Brown, A. Csótó, and R. Sherr, Nucl. Phys. A597, 66 (1996).

[7] H. Esbensen and G. F. Bertsch, Nucl. Phys. A600, 37 (1996); S. Typel, H. H. Wolter and G. Baur, Nucl. Phys. A613, 147 (1997).

[8] L. V. Grigorenko, B. V. Danilin, V. D. Efros, N. B. Shul'gina, and M. V. Zhukov, Phys. Rev. C 57, R2099 (1998); Phys. Rev. C, in press.

[9] K. Bennaceur, F. Nowacki, J. Okolowicz, and M. Ploszajczak, J. Phys. G 24, 1631 (1998); Nucl. Phys. A651, 289 (1999).

[10] A. Csótó, K. Langanke, S. E. Koonin, and T. D. Shoppa, Phys. Rev. C 52, 1130 (1995); A. Csótó, Phys. Lett. B 394, 247 (1997); A. Csótó and K. Langanke, Nucl. Phys. A636, 240 (1998).

[11] P. Descouvemont and D. Baye, Phys. Rev. C 60, 015803 (1999).

[12] F. C. Barker, Nucl. Phys. A588, 693 (1995).

[13] B. K. Jennings, S. Karataglidis, and T. D. Shoppa, Phys. Rev. C 58, 3711 (1998).

[14] H. M. Xu, C. A. Gagliargi, R. E. Tribble, A. M. Mukhamedzhnov, and N. K. Timofeyuk, Phys. Rev. Lett. 73, 2027 (1994).

[15] N. K. Timofeyuk, D. Baye, and P. Descouvemont, Nucl. Phys. A620, 29 (1997); N. K. Timofeyuk, Nucl. Phys. A632, 19 (1998).

[16] E. G. Adelberger et al., Rev. Mod. Phys. 70, 1265 (1998).

[17] A. M. Mukhamedzhanov and N. K. Timofeyuk, JETP Lett. 51, 282 (1990); Sov. J. Nucl. Phys. 51, 431 (1990). 
[18] A. Csótó, Heavy Ion Phys. 6, 103 (1997) nuclth/9704053; nucl-th/9712033.

[19] K. Varga and R. G. Lovas, Phys. Rev. C 43, 1201 (1991).

[20] A. Csótó and R. G. Lovas, Phys. Rev. C 46, 576 (1992).

[21] F. C. Barker, Aust. J. Phys. 33, 177 (1980).

[22] A. E. L. Dieperink and T. de Forest, Phys. Rev. C 10, 543 (1974).
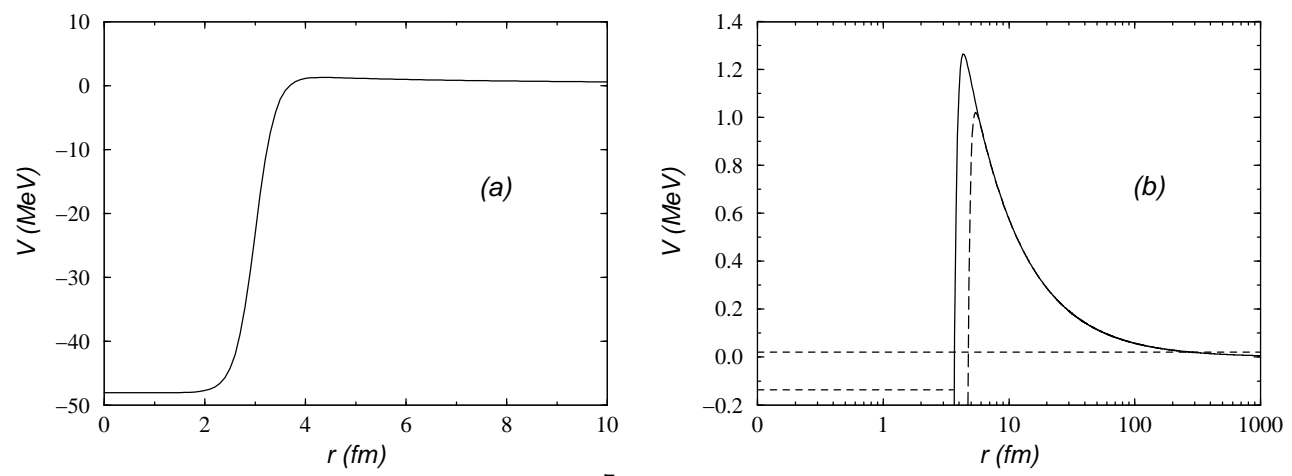

FIG. 1. Schematic local potential between ${ }^{7} \mathrm{Be}$ and $p$ (a), and its barrier region magnified (b). The horizontal dashed lines denote the energy of a $20 \mathrm{keV}$ proton incident on ${ }^{7} \mathrm{Be}$ and the binding energy, $-137 \mathrm{keV}$, of ${ }^{8} \mathrm{~B}$, relative to the ${ }^{7} \mathrm{Be}+p$ threshold, respectively. The long-dashed line shows the Coulomb barrier of a potential with a somewhat larger radius.

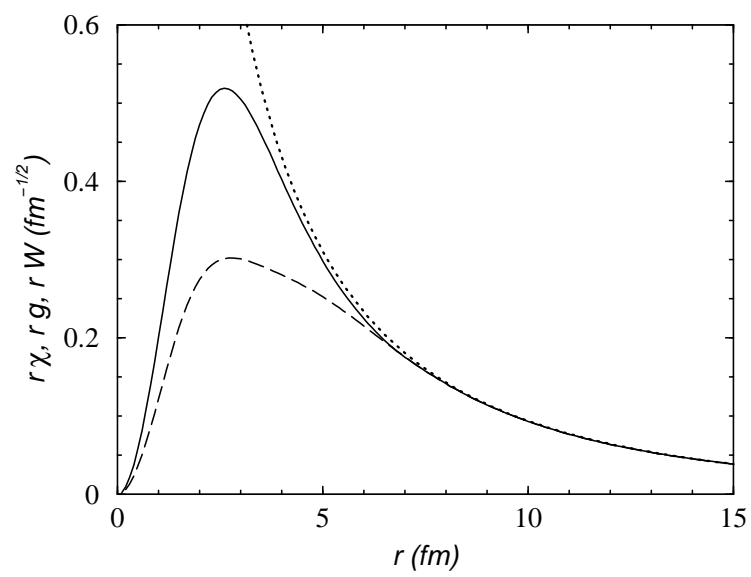

FIG. 2. The reduced ${ }^{7} \mathrm{Be}+p$ relative motion wave function ( $r \chi$, dashed line) and spectroscopic amplitude $(r g$, solid line) of ${ }^{8} \mathrm{~B}$ in the $I=2$ channel, coming from the microscopic cluster model of Refs. 10]. The dotted line is the corresponding ${ }^{7} \mathrm{Be}+p$ Coulomb-Whittaker function multiplied by the asymptotic normalization constant, $\bar{c}_{2}=0.763$. 